\title{
BMJ Open Factors critical to implementation success of cleaner cooking interventions in low- income and middle-income countries: protocol for an umbrella review
}

\author{
Esther A Boudewijns (D) , ${ }^{1}$ Debbie Vermond, ${ }^{2}$ Rianne M J J van der Kleij, ${ }^{2}$ \\ Niels H Chavannes, ${ }^{2}$ Onno C P van Schayck, ${ }^{1}$ Bruce Kirenga, ${ }^{3}$ \\ Evelyn A Brakema (iD ${ }^{2}$
}

To cite: Boudewijns EA, Vermond $D$, van der Kleij RMJJ, et al. Factors critical to implementation success of cleaner cooking interventions in low-income and middleincome countries: protocol for an umbrella review. BMJ Open 2020;10:e041821. doi:10.1136/ bmjopen-2020-041821

- Prepublication history and supplemental material for this paper is available online. To view these files, please visit the journal online (http://dx.doi org/10.1136/bmjopen-2020041821).

Received 19 June 2020 Revised 10 November 2020 Accepted 20 November 2020

Check for updates

(c) Author(s) (or their employer(s)) 2020. Re-use permitted under CC BY-NC. No commercial re-use. See rights and permissions. Published by BMJ.

${ }^{1}$ Department of Family Medicine, Care and Public Health Research Institute (CAPHRI), Maastricht University, Maastricht, The Netherlands

${ }^{2}$ Department of Public Health and Primary Care, Leiden University Medical Center, Leiden, The Netherlands ${ }^{3}$ Department of Medicine and Makerere Lung Institute, Makerere University, Kampala, Uganda

Correspondence to Esther A Boudewijns; esther.boudewijns@ maastrichtuniversity.nl

\section{ABSTRACT}

Introduction Over a third of the world's population relies on solid fuels as their primary energy source. These fuels have damaging effects on health, air quality and forest resources. Interventions to promote access to cleaner solid fuel cookstoves and clean fuels have existed for decades. However, the adoption by local communities has largely failed, which led to a waste of resources and suboptimal outcomes. Therefore, the objective of this umbrella review is to identify factors that determine implementation success for cleaner cooking interventions in low-resource settings and weigh their level of confidence in the evidence.

Methods and analysis We identified systematic and narrative reviews examining factors that influence the acquisition, initial adoption or sustained use of cleaner solid fuel cookstoves and clean fuels at any scale by a literature search in PubMed, Embase, Global Health Database, Cochrane, PsycINFO, Emcare, Web of Science and CINAHL, without date or language restrictions. The search was conducted on 23 0ctober 2017 and updated on 10 July 2019. Reviews based on qualitative, quantitative or mixed-methods studies were included and will be appraised using the Meta Quality Appraisal Tool combined with the Assessment of Multiple Systematic Reviews. Data will be extracted and factors affecting implementation will be coded using the Consolidated Framework for Implementation Research. The Grading of Recommendations Assessment, Development and Evaluation-Confidence in the Evidence from Reviews of Qualitative Research tool will be used to determine the level of confidence in the coded factors. Two researchers will independently conduct these steps.

Ethics and dissemination This umbrella review does not require the approval of an ethical review board. Study results will be published in an international peer-reviewed journal. The outcomes will be converted into two practical tools: one for cleaner solid fuel cookstoves and one for clean fuels. These tools can guide the development of evidence-based implementation strategies for cleaner cooking interventions in low-income and middle-income countries to improve implementation success. These tools should be pilot-tested and promoted among regional and global initiatives.

PROSPERO registration number CRD42018088687.

\section{Strengths and limitations of this study}

- This umbrella review will aggregate up-to-date evidence on factors critical to implementation success for cleaner cooking interventions in low-resource settings and weigh the level of confidence in the evidence.

- We will comprehensively search eight databases without date or language restrictions, and two researchers will independently apply validated tools throughout each step of the process.

- Narrative reviews will also be included in the study, as only a fraction of the literature has been reported in systematic reviews; the lower methodological rigour of narrative reviews (compared with systematic reviews) will be considered during appraisal of the methodological quality of the reviews.

- The identified implementation factors will be integrated into two hands-on implementation tools to facilitate the design of evidence-based implementation strategies for cleaner cooking interventions and hence stimulate the translation of evidence into practice.

\section{INTRODUCTION}

The use of solid fuels for cooking is a deeprooted practice for approximately 2.8 billion people, especially in low-income and middle-income countries (LMICs). ${ }^{1}$ Solid fuels include biomass fuels (such as wood, dung, crop residues and charcoal) and coal. Combustion of solid fuels leads to emission of greenhouse gases and black carbon, aggravating climate change. It also leads to high levels of household air pollution affecting health. ${ }^{2}$ Furthermore, collecting fuels leads to forest degradation and deforestation and is time consuming for women and children involved in fuel gathering. ${ }^{3}$ Interventions that promote the replacement of traditional cookstoves (that use solid fuels or kerosene as fuel) by cleaner solid fuel cookstoves 
(later referred to as cleaner cookstoves) or clean fuels seem to be promising solutions due to higher combustion efficiency and lower emissions. Clean fuels include liquefied petroleum gas (LPG), natural gas, alcohol fuels, biogas, solar cookers and electricity. ${ }^{4}$ Kerosene stoves are relatively efficient but are discouraged due to significant health risks. ${ }^{4}$ Cleaner cookstoves came on the market in the 1950s and were later supported by promising laboratory-based experiments with different models, trying to improve combustion efficiency. ${ }^{56}$ Subsequently, many non-governmental organisations and governments promoted the large-scale rollout of these stoves. The push for a transition to clean fuels has come into force since World Health Organisation (WHO) published guidelines for indoor air quality and household fuel combustion. ${ }^{7}$ However, real-world outcomes of interventions that promoted the switch to cleaner cookstoves or clean fuels have often not been fruitful. ${ }^{56}$ Many of the programmes ran into hurdles and failed ${ }^{8}$; adoption rates are often not reported in the literature, and if they are, rates may vary between $4 \%$ and $10 \% .^{9-13}$ Moreover, even if cleaner stoves and clean fuels are used in local communities, they are often combined with traditional ones. ${ }^{14}$ Amidst scarce resources, implementation efforts have thus largely been a waste of time, money, energy and human resource. Progress in large-scale implementation has been inexplicably slow. ${ }^{315}$

Implementation, the act of carrying an intervention into widespread use, is a complex process. ${ }^{16}$ Although the failure to implement cleaner cooking interventions has been reported already in the 1980s and 1990s, ${ }^{813} 17-19$ implementation strategies and adoption rates have generally not changed accordingly. Numerous additional studies have since then examined barriers and facilitators for the implementation of cleaner cooking interventions, including more than a dozen systematic and narrative reviews. Although evidence has been consolidated in a study by Puzzolo $e t a l,{ }^{20}$ an up-to-date overview of the evidence, including more recently published reviews, is missing. Furthermore, evidence of factors that influence the implementation of clean fuels was limited in this study. Therefore, we will conduct an umbrella review or in other words a review of systematic and narrative reviews. We aim to systematically identify critical factors for implementation success of cleaner cooking interventions in LMICs and to weigh their level of evidence. We define implementation success as sustained predominant use of cleaner cookstoves and clean fuels. ${ }^{21}$ As we expect limited evidence on sustained use (defined as use for 1-2years after acquisition or use for more than 2 years $^{20}$ ), we will also include factors that relate to acquisition (defined as purchase or installation) and initial adoption (defined as use for less than 1 year from acquisition ${ }^{20}$ ) of cleaner cooking interventions. Findings will be consolidated in two easy-to-use implementation tools: one for cleaner cookstoves and one for clean fuels. These tools can practically guide the development of evidence-based implementation strategies. Use of these tools could ensure more effective use of scarce resources and, ultimately, alleviate climate change and improve health outcomes.

\section{METHODS AND ANALYSIS}

This study is part of a larger study conducted by Brakema et $a l^{22}$ This latter study focused on critical implementation factors for interventions targeting chronic lung disease in LMICs, as part of the Horizon 2020 Free Respiratory Evaluation and Smoke-exposure reduction by primary Health cAre Integrated gRoups (FRESH AIR) project. ${ }^{23}$ Because the search resulted in a high number of articles that focus on the implementation of cleaner cooking interventions, it was decided to split the review into two parts. The review of Brakema et al includes articles on implementation of all interventions other than cleaner cooking interventions (such as tobacco cessation or the implementation of guidelines), while the current study is an umbrella review that includes reviews focusing on cleaner cooking interventions. The study is registered at PROSPERO (CRD42018088687). Because the methodology for this umbrella review differs from the methodology used in the review by Brakema $e t a l,{ }^{22}$ and because an umbrella review of systematic and narrative reviews is not common, ${ }^{245}$ it was decided to record the protocol for this study to increase transparency.

\section{Search strategy and inclusion criteria}

The initial search was part of the study conducted by Brakema et al..$^{22}$ The search strategy was developed together with a certified medical librarian. Search terms included (synonyms of) implementation, LMICs and interventions targeting chronic lung health, including cleaner cooking interventions. No names of fuels or specific cooking interventions, such as LPG or biogas, were included in the search strategy. This is a limitation of the study. Cleaner cooking interventions were included regardless whether they were defined in terms of health benefits. The search terms are displayed in online supplemental material S1. Searches were conducted using the databases PubMed, Embase, Global Health Database, Cochrane, PsycINFO, Emcare, Web of Science and CINAHL, with no date or language restrictions. The search was conducted on 23 October 2017 and updated on 10 July 2019. The full search strategy and the operationalisation is described elsewhere. ${ }^{22}$ Furthermore, experts in the field of cleaner cooking interventions will be consulted to identify relevant publications from July 2019. All reviews regarding the implementation of cleaner cookstoves and clean fuels in LMICs were extracted from the results and will be used in the current study. Given the expected large number of cookstove studies covered in reviews other than systematic reviews (narrative reviews), it was decided to be inclusive and not limit the search to systematic reviews only. Reviews based on quantitative, qualitative or mixedmethods studies will be considered relevant. ${ }^{16}$ Articles will be excluded if they focus on legislation at a national governmental level or on hypothetical interventions. 


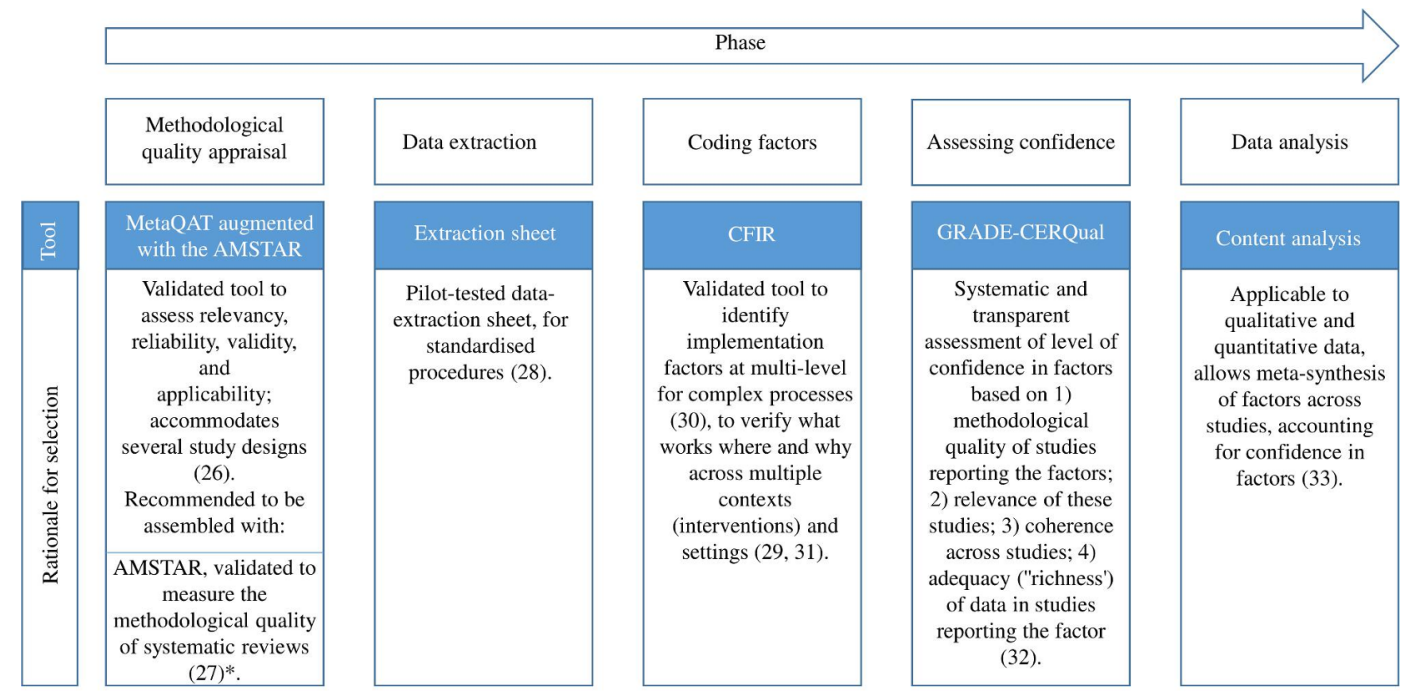

Figure 1 Tools that will be used in each phase. Adapted from Brakema et al. ${ }^{22}$ Tools to assess the quality of narrative reviews are not in the list of recommended design-specific companion tools to augment the MetaQAT tool. ${ }^{26}$ Therefore, it was decided to use the AMSTAR-tool for systematic and narrative reviews. AMSTAR, Assessment of Multiple Systematic Reviews; CFIR, Consolidated Framework for Implementation Research; GRADE-CERQual, Grading of Recommendations Assessment, Development and Evaluation-Confidence in the Evidence from Reviews of Qualitative research; MetaQat, Meta Quality Appraisal Tool.

Besides, articles will be excluded if no factors that influence implementation are reported or if the full text is not available after contacting the authors.

The steps that will be conducted, and the tools used to conduct these steps, are displayed in figure 1. Methodological quality assessment, data extraction and data analysis will be conducted in the same manner for systematic and narrative reviews. The risk of biased recommendations in narrative reviews will be accounted for during appraisal of the methodological quality of the reviews.

\section{Methodological quality assessment}

The Meta Quality Appraisal Tool (MetaQAT) ${ }^{26}$ combined with the Assessment of Multiple Systematic Reviews $(\text { AMSTAR })^{27}$ will be used to assess the methodological quality of included studies. The MetaQAT assesses the relevancy, reliability, validity and applicability of the included studies. ${ }^{26}$ The AMSTAR will be embedded into the MetaQAT, as recommended by the MetaQAT authors. ${ }^{26}$ The results will be used as input for the assessment of confidence in the evidence.

\section{Data extraction}

Data will be extracted using standardised pilot-tested extraction forms. ${ }^{28}$ We will extract data on descriptive characteristics (author; year; narrative/systematic review; whether the review included quantitative, qualitative or mixed-method studies; country; setting/population; fuel used before intervention; intervention targeting cleaner cookstoves vs clean fuels (type); outcome; data sources; funding source of the review; role of funding organisation) and implementation factors. Factors will not be extracted if they are speculations.

\section{Data analysis}

Content analysis will be used for data analysis. ${ }^{29}$ Content analysis is a research technique for making replicable and valid inferences from data to their context. ${ }^{30}$ It can be used to synthesise qualitative and quantitative data. ${ }^{29}$ First, to prepare the data for content analysis, the implementation factors will be coded using the Consolidated Framework for Implementation Research (CFIR). ${ }^{31}$ The CFIR is validated for identifying implementation factors at multilevel for complex processes ${ }^{32}$ and helps to understand what works where and why across multiple contexts (interventions) and settings. ${ }^{31} 33$ Implementation factors are categorised in five domains: (1) intervention characteristics, (2) outer setting, (3) inner setting, (4) characteristics of individuals involved and (5) the implementation process. ${ }^{31}$ Each CFIR domain includes several constructs. Several constructs were added to the CFIR by Brakema et $a l^{22}$ such as the extent to which language affects the implementation process. If relevant, more constructs will be added inductively to the framework. Second, the Grading of Recommendations Assessment, Development and Evaluation-Confidence in the Evidence from Reviews of Qualitative research (GRADE-CERQual) will be used to assess the confidence in the included factors. ${ }^{34}$ The GRADE-CERQual consists of four domains: methodological limitations, relevance, coherence and adequacy. However, coherence will not be included as this will be taken into account in the end by counting the number of studies that mention the factor. For each of the other three domains, a score will be assigned per study in which the factor appeared. The score for adequacy will be determined based on the richness of the data. Relevance and methodological limitations will be scored on 
the aforementioned MetaQAT-AMSTAR tool (relevance and reliability+validity, respectively). This score ranges from one point (substantial concerns) to four points (no to very minor concerns). Third, an overall level of confidence in the factor will be determined by totalling the scores of the three domains. This total score will thus be higher if the studies' adequacy, relevance or quality of the methodology was appraised as high or if the factor appeared in more studies (the principle of content analysis). Guidance to preclude the double-counting that overstates the evidence due to overlap among reviews is limited and inconsistent. ${ }^{35}$ To gain insight into the frequency of double-counting, a matrix of the primary studies included in each review will be presented. ${ }^{36}$

At least two authors will independently conduct the screening and selection process, the data extraction and the quality appraisal of the included studies. A third researcher will solve any discrepancies. Endnote will be used to manage records and data throughout the review. In case of deviations from the protocol, these amendments will be described in the final paper.

\section{Patient and public involvement}

Patients and the public will not be involved in this research.

\section{Ethics and dissemination}

This umbrella review does not require the approval of an ethical review board. Study results will be published in an international peer-reviewed journal. The outcomes will be converted in two practical tools, one for cleaner cookstoves and one for clean fuels, that can guide the development of evidence-based implementation strategies for cleaner cooking interventions in LMICs. These tools should be pilot-tested in future cookstove implementation projects and adapted accordingly. Furthermore, the tools should be presented to brokers in large networks, including the Clean Cooking Implementation Science Network, the Clean Cooking Alliance, and WHO. We have already reached out to several of these organisations. Ideally, these organisations should then promote the use of the tool to make it well known and easily available.

\section{DISCUSSION}

With this umbrella review, we aim to consolidate all available evidence on critical factors for successful implementation of cleaner cookstoves and clean fuels in LMICs and weigh the level of confidence in the evidence. Implementation methodologies are considered as main priorities in implementation science, especially in low-resource settings. ${ }^{37-39}$ This umbrella review uses a comprehensive implementation science framework, includes up-to-date data from both systematic and narrative reviews and will result in two practical tools that may aid in the development of evidence-based implementation strategies. An example of such a tool can be found in the study of Brakema et al. ${ }^{22}$ These tools should help to develop evidence-based implementation strategies for cleaner cooking interventions in the field.

With the increasing number of scientific reviews comes a need for third-level analyses, combining evidence of multiple reviews. ${ }^{40}$ Various terms are given to these overviews of reviews, including meta-reviews, review of systematic reviews, reviews of reviews or umbrella reviews. ${ }^{41}$ A precise definition of the different terms is unclear. However, the common denominator is that they often only assess systematic reviews. Umbrella reviews are usually more inclusive of different types of systematic reviews compared with the other overview of reviews. ${ }^{41} \mathrm{We}$ decided to also include narrative reviews in this umbrella review given the nature of the sector. However, narrative reviews may increase the risk of biased recommendations as they have less stringent criteria for information selection and appraisal than systematic reviews. ${ }^{42}$ We will account for this risk of bias as the level of confidence in the evidence is based on the quality of the methodology of the review in which the factor is reported. Excluding narrative reviews would exclude a considerable amount of available evidence. We decided not to distinguish between factors influencing adoption and factors influencing sustained use, as we expect limited evidence on sustained use only. Furthermore, in cleaner cooking literature, adoption and sustained use are often not clearly distinguished. ${ }^{43}$ Ideally, studies would investigate the outcomes of cleaner cooking interventions over the course of several years, as the outcomes are known to differ over time. ${ }^{44}$ Notably, we will weigh confidence in the factors that influence the implementation of cleaner cookstoves and clean fuels, based on the frequency of occurrence in the studies, and the quality of the study in which the factors are reported. This should not be interpreted as weighing or prioritising the importance of the factors. ${ }^{45} 46$ We also decided not to separately report on barriers and facilitators, as reversed barriers will most likely serve as facilitators and vice versa. ${ }^{2246}$ We decided to include both cleaner cookstoves and clean fuels in this review, although the first are often not classified as 'clean' according to the guidelines of WHO due to high exposure levels. ${ }^{4}$ However, cleaner cookstoves can play an important role in the transition to clean fuels and are therefore included in this review. Furthermore, newer cleaner cookstoves, such as advanced biomass cookstoves, will be included in this umbrella review, but it is likely that these will not be covered by the identified reviews. However, findings of the other cleaner cookstoves are probably largely applicable to the newer cleaner cookstoves. ${ }^{20}$

This study will result in two tools with an overview of implementation factors, confidence in the evidence of the factors and practical examples for implementers to promote large-scale uptake of cleaner cookstoves and clean fuels, respectively. These tools can therefore complement WHO Clean Household Energy Solutions Toolkit. ${ }^{47}$ Successful implementation, including sustained use, of cleaner cooking interventions could ultimately alleviate climate change and improve health outcomes. ${ }^{48}$ 
Acknowledgements We would like to thank J Rosenthal for critically reading the manuscript and providing suggestions to improve this protocol. We would like to acknowledge Metamorfose Vertalingen BV for their assistance in proofreading the manuscript.

Contributors EvAB, DV, RMJJvdK, NHC and BK are part of the Free Respiratory Evaluation and Smoke-exposure reduction by primary Health cAre Integrated gRoups (FRESH AIR) project and were involved in the study of Brakema et al, which included implementation of all interventions other than cleaner cooking interventions. EvAB initiated the idea to start a review of reviews on cleaner cooking interventions, and together with EsAB elaborated the concept and design. EsAB wrote the first version of the manuscript. EvAB supervised the various stages of the writing of the manuscript. DV, RMJJvdK, NHC, BK and OCPvS critically reviewed the paper. All authors have read and approved the final version of the manuscript.

Funding The study stems from the FRESH AIR project, funded by the EU Research and Innovation program Horizon2020 (Health, Medical research and the challenge of ageing) under grant agreement no. 680997.

Disclaimer The funders had no role in the study design.

Competing interests None declared.

Patient consent for publication Not required.

Provenance and peer review Not commissioned; externally peer reviewed.

Supplemental material This content has been supplied by the author(s). It has not been vetted by BMJ Publishing Group Limited (BMJ) and may not have been peer-reviewed. Any opinions or recommendations discussed are solely those of the author(s) and are not endorsed by BMJ. BMJ disclaims all liability and responsibility arising from any reliance placed on the content. Where the content includes any translated material, BMJ does not warrant the accuracy and reliability of the translations (including but not limited to local regulations, clinical guidelines, terminology, drug names and drug dosages), and is not responsible for any error and/or omissions arising from translation and adaptation or otherwise.

Open access This is an open access article distributed in accordance with the Creative Commons Attribution Non Commercial (CC BY-NC 4.0) license, which permits others to distribute, remix, adapt, build upon this work non-commercially, and license their derivative works on different terms, provided the original work is properly cited, appropriate credit is given, any changes made indicated, and the use is non-commercial. See: http://creativecommons.org/licenses/by-nc/4.0/.

\section{ORCID iDs}

Esther A Boudewijns http://orcid.org/0000-0001-9087-1712

Evelyn A Brakema http://orcid.org/0000-0002-7376-4648

\section{REFERENCES}

1 Sood A, Assad NA, Barnes PJ, et al. ERS/ATS workshop report on respiratory health effects of household air pollution. Eur Respir $J$ 2018;51. doi:10.1183/13993003.00698-2017. [Epub ahead of print: 04 Apr 2018]

2 Onakomaiya D, Gyamfi J, Iwelunmor J, et al. Implementation of clean cookstove interventions and its effects on blood pressure in lowincome and middle-income countries: systematic review. BMJ Open 2019:9:e026517.

3 Jeuland MA, Pattanayak SK. Benefits and costs of improved cookstoves: assessing the implications of variability in health, forest and climate impacts. PLoS One 2012;7:e30338.

4 World Health Organization. WHO guidelines for indoor air quality: household fuel combustion. Geneva, Switserland: World Health Organization, 2014.

5 Thomas E, Wickramasinghe K, Mendis S, et al. Improved stove interventions to reduce household air pollution in low and middle income countries: a descriptive systematic review. BMC Public Health 2015;15:650

6 Honkalaskar VH, Bhandarkar UV, Sohoni M. Development of a fue efficient cookstove through a participatory bottom-up approach. Energy Sustain Soc 2013;3:1-21.

7 World Health Organization. Fuel for life: household energy and health Geneva: World Health Organization, 2006.

8 Barnes DF, Openshaw K, Smith KR, et al. The design and diffusion of improved cooking stoves. World Bank Res Obs 1993;8:119-41.

9 Bensch G, Grimm M, Peters J. Why do households forego high returns from technology adoption? Evidence from improved cooking stoves in Burkina Faso. J Econ Behav Organ 2015;116:187-205.
10 Clark S, Carter E, Shan M, et al. Adoption and use of a semi-gasifier cooking and water heating stove and fuel intervention in the Tibetan Plateau, China. Environ Res Lett 2017;12:075004.

11 El Tayeb Muneer S, Mukhtar Mohamed EW, Muneer SET. Adoption of biomass improved cookstoves in a patriarchal Society: an example from Sudan. Sci Total Environ 2003;307:259-66.

12 Jagger $\mathrm{P}$, Jumbe $\mathrm{C}$. Stoves or sugar? willingness to adopt improved Cookstoves in Malawi. Energy Policy 2016;92:409-19.

13 Manibog FR. Improved cooking stoves in developing countries: problems and opportunities. Annu Rev Energy 1984;9:199-227.

14 Ruiz-Mercado I, Masera O. Patterns of stove use in the context of fuel-device stacking: rationale and implications. Ecohealth 2015;12:42-56.

15 Brakema EA, van der Kleij RM, Vermond D, et al. Let's stop dumping cookstoves in local communities. It's time to get implementation right. NPJ Prim Care Respir Med 2020;30.

16 Peters DH, Adam T, Alonge O, et al. Implementation research: what it is and how to do it. Br Med J 2013;347:f6753.

17 Hyman EL. The strategy of production and distribution of improved charcoal stoves in Kenya. World Dev 1987;15:375-86.

18 Mannan M. Women targeted and women negated. Dev Pract 1996;6:113-20.

19 Pandey S, Yadama GN. Community development programs in Nepal: a test of diffusion of innovation theory. Soc Serv Rev 1992;66:582-97.

20 Puzzolo E, Stanistreet D, Pope D, et al. Factors influencing the largescale uptake by households of cleaner and more efficient household energy technologies. A systematic review. London: EPPI-Centre, University of Londen, 2013.

21 Rosenthal J, Balakrishnan K, Bruce N, et al. Implementation science to accelerate clean cooking for public health. Environ Health Perspect 2017;125:A3-7.

22 Brakema EA, Vermond D, Pinnock H, et al. Implementing lung health interventions in low- and middle-income countries: a fresh air systematic review and meta-synthesis. Eur Respir J 2020;56. doi:10.1183/13993003.00127-2020. [Epub ahead of print: 23 Jul 2020].

23 Cragg L, Williams S, Chavannes NH. Fresh air: an implementation research project funded through horizon 2020 exploring the prevention, diagnosis and treatment of chronic respiratory diseases in low-resource settings. NPJ Prim Care Respir Med 2016:26:1-5.

24 San-Juan-Rodriguez A, Newman TV, Hernandez I, et al. Impact of community pharmacist-provided preventive services on clinical, utilization, and economic outcomes: an umbrella review. Prev Med 2018;115:145-55.

25 Posadzki PP, Bajpai R, Kyaw BM, et al. Melatonin and health: an umbrella review of health outcomes and biological mechanisms of action. BMC Med 2018;16:18.

26 Rosella L, Bowman C, Pach B, et al. The development and validation of a meta-tool for quality appraisal of public health evidence: meta quality appraisal tool (MetaQAT). Public Health 2016;136:57-65.

27 Shea BJ, Grimshaw JM, Wells GA, et al. Development of AMSTAR: a measurement tool to assess the methodological quality of systematic reviews. BMC Med Res Methodol 2007;7:10.

28 Higgins JP, Thomas J, Chandler J, et al. Cochrane Handbook for systematic reviews of interventions, version 6 . John Wiley \& Sons, 2019. https://training.cochrane.org/handbook/current

29 Dixon-Woods M, Agarwal S, Young B, et al. Integrative approaches to qualitative and quantitative evidence. London: Health Development Agency, 2004: 181

30 Krippendorff K. Content analysis: an introduction to its methodology. Sage publications, 2018

31 Damschroder LJ, Aron DC, Keith RE, et al. Fostering implementation of health services research findings into practice: a consolidated framework for advancing implementation science. Implement Sci 2009;4:1-15.

32 Damschroder LJ, Lowery JC. Evaluation of a large-scale weight management program using the consolidated framework for implementation research (CFIR). Implement Sci 2013;8:51.

33 Moullin JC, Sabater-Hernández D, Fernandez-Llimos F, et al. A systematic review of implementation frameworks of innovations in healthcare and resulting generic implementation framework. Health Res Policy Syst 2015;13:16.

34 Lewin S, Glenton C, Munthe-Kaas H, et al. Using qualitative evidence in decision making for health and social interventions: an approach to assess confidence in findings from qualitative evidence syntheses (GRADE-CERQual). PLoS Med 2015;12:e1001895.

35 Pollock M, Fernandes RM, Becker LA, et al. What guidance is available for researchers conducting overviews of reviews of healthcare interventions? A scoping review and qualitative metasummary. Syst Rev 2016;5:190. 
36 Lunny C, Brennan SE, Reid J, et al. Overviews of reviews incompletely report methods for handling overlapping, discordant, and problematic data. J Clin Epidemiol 2020;118:69-85.

37 Ridde V. Need for more and better implementation science in global health. BMJ Glob Health 2016;1:e000115.

38 Waltz TJ, Powell BJ, Fernández ME, et al. Choosing implementation strategies to address contextual barriers: diversity in recommendations and future directions. Implement Sci 2019;14:1-15.

39 Siddiqi K, Newell J, Robinson M. Getting evidence into practice: what works in developing countries? Int J Qual Health Care 2005;17:447-54.

40 Tsagris M, Fragkos KC. Umbrella reviews, overviews of reviews, and meta-epidemiologic studies: similarities and differences. Umbrella reviews: Springer, Cham, 2016: 43-54.

41 Petrovskaya O, Lau F, Antonio M. Synthesising evidence on patient portals: a protocol for an umbrella review. BMJ Open 2019;9:e024469.

42 Bollini P, Pampallona S, Kupelnick B, et al. Improving compliance in depression: a systematic review of narrative reviews. J Clin Pharm Ther 2006;31:253-60.
43 Debbi S, Elisa P, Nigel B, et al. Factors influencing household uptake of improved solid fuel stoves in low- and middle-income countries: a qualitative systematic review. Int J Environ Res Public Health 2014;11:8228-50.

44 Hanna R, Duflo E, Greenstone M. Up in smoke: the influence of household behavior on the long-run impact of improved cooking stoves. Am Econ J Econ Policy 2016;8:80-114.

45 Puzzolo E, Pope D, Stanistreet D, et al. Clean fuels for resource-poor settings: a systematic review of barriers and enablers to adoption and sustained use. Environ Res 2016;146:218-34.

46 Rehfuess EA, Puzzolo E, Stanistreet D, et al. Enablers and barriers to large-scale uptake of improved solid fuel stoves: a systematic review. Environ Health Perspect 2014;122:120-30.

47 World Health Organization. Clean household energy solutions toolkit (chest). Available: https://www.who.int/airpollution/household/chest/ en/

48 Energy Sector Management Assistance Program (ESMAP). The state of access to modern energy cooking services. Washington, DC: World Bank, 2020. 